\title{
The continuum intensity as a function of magnetic field
}

\section{Local magnetic flux and convective flows}

\author{
P. Kobel ${ }^{1,2}$, S. K. Solanki ${ }^{1,3}$, and J. M. Borrero ${ }^{4}$ \\ 1 Max-Planck Institut für Sonnensystemforschung, Max-Planck-Straße 2, 37191 Katlenburg-Lindau, Germany \\ e-mail: philippe.kobel@a3.epfl.ch \\ 2 EPFL, Laboratoire des Machines Hydrauliques, 1007 Lausanne, Switzerland \\ 3 School of Space Research, Kyung Hee University, Yongin, 446-701 Gyeonggi, Korea \\ ${ }^{4}$ Kiepenheuer-Institut für Sonnenphysik, Schöneckstr. 6, 79104 Freiburg, Germany \\ Received 18 October 2011 / Accepted 6 February 2012
}

ABSTRACT

\begin{abstract}
Context. To deepen our understanding of the role of small-scale magnetic fields in active regions (ARs) and in the quiet Sun (QS) on the solar irradiance, it is fundamental to investigate the physical processes underlying their continuum brightness. Previous results showed that magnetic elements in the QS reach larger continuum intensities than in ARs at disk center, but left this difference unexplained.

Aims. We use Hinode/SP disk center data to study the influence of the local amount of magnetic flux on the vigour of the convective flows and the continuum intensity contrasts.

Methods. The apparent (i.e. averaged over a pixel) longitudinal field strength and line-of-sight (LOS) plasma velocity were retrieved by means of Milne-Eddington inversions (VFISV code). We analyzed a series of boxes taken over AR plages and the QS, to determine how the continuum intensity contrast of magnetic elements, the amplitude of the vertical flows and the box-averaged contrast were affected by the mean longitudinal field strength in the box (which scales with the total unsigned flux in the box).

Results. Both the continuum brightness of the magnetic elements and the dispersion of the LOS velocities anti-correlate with the mean longitudinal field strength. This can be attributed to the "magnetic patches" (here defined as areas where the longitudinal field strength is above $100 \mathrm{G}$ ) carrying most of the flux in the boxes. There the velocity amplitude and the spatial scale of convection are reduced. Due to this hampered convective transport, these patches appear darker than their surroundings. Consequently, the average brightness of a box decreases as the the patches occupy a larger fraction of it and the amount of embedded flux thereby increases. Conclusions. Our results suggest that as the magnetic flux increases locally (e.g. from weak network to strong plage), the heating of the magnetic elements is reduced by the intermediate of a more suppressed convective energy transport within the larger and stronger magnetic patches. This, together with the known presence of larger magnetic features, could explain the previously found lower contrasts of the brightest magnetic elements in ARs compared to the QS. The inhibition of convection also affects the average continuum brightness of a photospheric region, so that at disk center, an area of photosphere in strong network or plage appears darker than a purely quiet one. This is qualitatively consistent with the predictions of 3D MHD simulations.
\end{abstract}

Key words. Sun: activity - Sun: photosphere - Sun: surface magnetism - Sun: granulation - Sun: faculae, plages

\section{Introduction}

Variations of the total solar irradiance (TSI) on timescales from days to the solar cycle can be reasonably well reproduced based on the evolving distribution of solar surface magnetic fields (which is the basis of e.g. the SATIRE reconstructions, Fligge et al. 2000; Solanki \& Fligge 2002; Krivova et al. 2003; Wenzler et al. 2006). Although the TSI reconstructions proved very successful (with a correlation of up to 0.98 with the measured irradiance, Ball et al. 2011), they are based on an empirical relationship between the emergent intensity of a pixel and the measured magnetic field (via MDI magnetograms) whose simple linearity lacks physical basis; its justification relies on its ability to reproduce the disk-average irradiance and the convenience of using a single model atmosphere for all faculae (for more details see Fligge et al. 2000). In particular, the spectral radiance of a given pixel is assumed to be uniquely determined by the magnetic signal at that pixel, thus neglecting its magnetic environment and location on the Sun.

The surface magnetic field outside Sunspots is mainly distributed in active region (AR) plages and in the quiet Sun (QS) network outlining supergranular cells. There the magnetic flux is often concentrated into features traditionally described in terms of flux tubes, with field strengths on the order of $\mathrm{kG}$ (e.g. Frazier \& Stenflo 1972; Stenflo 1973; Rabin 1992; Rüedi et al. 1992) and a spectrum of sizes (see Solanki et al. 2006, for a review). At the lower end of this size spectrum are found the socalled "magnetic elements" (see Schüssler 1992; Solanki 1993), which have been spatially resolved recently by the balloon-borne SUNRISE ImaX observations at an angular resolution of $\sim 0$ " 14 (Lagg et al. 2010). As a result of being hotter than their surroundings at equal optical depth (Schüssler 1992), they often appear brighter than the average quiet photosphere, i.e. they have a positive "contrast". This is particularly pronounced when observing in the core of spectral lines and in molecular bands (Chapman \& Sheeley 1968; Sheeley 1969; Muller \& Roudier 1984), but also holds at continuum wavelengths or broader wavelength bands, even at disk center, if the magnetic elements are sufficiently resolved (see Muller \& Keil 1983; Foukal \& Fowler 1984; Lawrence 1988; Riethmüller et al. 2010; Schüssler \& Solanki 1988; Vögler \& Schüssler 2003, the two last citations refering to radiation-MHD simulations). Note that the contrast 
of magnetic features further increases from the disk center to the limb in a way that is still a matter of debate (Steiner 2007) and shall be treated in a forthcoming paper. Therefore, taking into account the larger area coverage of magnetic elements compared to sunspots, their radiance overcompensates the solar brightness deficit due to sunspots at activity maximum (Fröhlich 2000). It is known that ARs typically harbor more magnetic flux per unit area than the network and thus a higher number density of magnetic elements. The larger available flux in ARs also leads to the formation of larger and darker magnetic features such as pores and micropores, the latter being small (sub-arcsec) magnetic features that are darker than the QS continuum (Beckers \& Schröter 1968; Tarbell \& Title 1977). The inner part of supergranulation cells, the so-called "internetwork" (IN), also contains small magnetic elements but in lower number density (Muller 1983; Lites 2002; Domínguez Cerdeña et al. 2003; de Wijn et al. 2005, 2008). Finally, the solar photosphere contains weaker equipartition fields everywhere (detected mostly in the IN by, e.g. Lin 1995; Solanki et al. 1996; Lites 2002; Khomenko et al. 2003), but the latter have been estimated to bring negligible contributions to the TSI variations (Schnerr \& Spruit 2011).

To determine how these different components of solar surface magnetism contribute to the solar irradiance, one should investigate the intensity-magnetic field relation within ARs, the QS network and even the IN. In the 1990's, Title et al. (1989); Topka et al. (1992); Lawrence et al. (1993) and Topka et al. (1997) performed a series of ground-based studies of continuum intensity vs. magnetogram signal in ARs and in the QS at disk center, and found that the QS continuum intensity contrast reaches larger values than in ARs at equal magnetogram signal. This early result was confirmed by our recent study using Hinode/SP data at a constant and higher spatial resolution than the earlier studies (Kobel et al. 2011, hereafter Paper I). Note that in our study, the contrast of magnetic elements could be interpreted as a measure of their intrinsic brightness by virtue of the comparable contrast references used in ARs and in the QS. We also found that the bright magnetic elements in ARs and in the QS share similar filling factors (along with similar $\mathrm{kG}$ field strengths and inclinations close to vertical). Assuming that, at Hinode's resolution and at disk center, the filling factor reflects the size of the unresolved magnetic elements (at the height of line formation), this questions the conventional interpretation that the brightness of magnetic features is primarily dictated by their size (Spruit \& Zwaan 1981, see also the Introduction of Paper I).

As discussed in Paper I, the other factor that could possibly influence the brightness of magnetic elements (besides their size) is the surrounding convective energy transport, because it determines the energy available to be radiated into the flux tubes through their "hot walls" (Spruit 1976). Since AR plages typically have a larger mean field strength than the QS network, the Lorentz force should inhibit the convective flows more strongly in ARs. The granulation indeed appears "abnormal" in plages (e.g. Dunn \& Zirker 1973), such that its spatial scale is reduced compared to the QS, as are both the vertical and horizontal components of the flow field (Schmidt et al. 1988; Title et al. 1989, 1992; Keller \& Koutchmy 1991). 3D MHD simulations also disclose a similar behavior as the mean field increases (Vögler 2005). Furthermore, these carefully set up simulations (with constant inflowing entropy density at the bottom boundary) demonstrated that the convective energy transport was increasingly inhibited to the point of altering the vertical radiative energy output from the boxes, thus providing an explanation for magnetic elements appearing darker in more active environments at disk center.

The work presented herein is an attempt to explain the effect of the local amount of magnetic flux on the continuum brightness of magnetic elements and on the local average continuum brightness of the solar surface at disk center. Inspired by MHD simulations, we carried out a "local box analysis" that considers a series of small square fields of view taken from different regions of QS and ARs (see Sect. 2.2) located at disk center, observed with the spectropolarimeter instrument onboard Hinode. In Sect. 3, we examine the relation between the contrast and the longitudinal field strength inside these boxes. In particular, we assess the influence of the mean longitudinal field strength in the box on the intensity contrast of the magnetic elements, on the vigour of the vertical convective flows, and on the contrast averaged over the box. One reason to restrict our study to disk center is that in this way we can interpret the Doppler velocities retrieved by Milne-Eddington inversions from the data (see Sect. 2.1) in terms of vertical flows. These results are then discussed in Sect. 4, with special emphasis on the role of the magnetically-suppressed convection on the contrasts.

\section{Dataset}

\subsection{Hinode/SP scans and inversions}

We used an ensemble of 6 spectropolarimetric scans over ARs and four scans over the QS performed very close to disk center (see Table 1) by the Hinode/SP instrument in its "normal mode", corresponding to an integration time of $4.8 \mathrm{~s}$ and rms polarimetric noise of $\sim 10^{-3}$ in units of the continuum intensity $I_{\mathrm{c}}$ (Tsuneta et al. 2008; Suematsu et al. 2008). Maps of $I_{\mathrm{c}}$ and $\mu$ values (calculated in the red continuum of the $630.2 \mathrm{~nm}$ line) were calculated by the sp_prep procedure. Note that we used the same scans as in Paper I.

The observed Stokes spectra at each pixel of the scans were inverted with the VFISV (very fast inversion of the stokes vector) Milne-Eddington code of Borrero et al. (2010) (we refer to that article for all details of the code). Unlike in Paper I, no filling factor treatment was considered here, as we were only interested in retrieving quantities averaged over a pixel ${ }^{1}$. In particular, we were concerned by the following inversion parameters: the lineof-sight (LOS) components of the plasma velocity, $v_{\text {los }}$, and the apparent strength of magnetic field $B_{\text {app,los }}=B|\cos \gamma|$, where $B$ is the field strength and $\gamma$ the inclination with respect to the LOS. Since $B$ is averaged over the pixel (and thus not "intrinsic"), this definition of $B_{\text {app,los }}$ is physically equivalent to the one used in Paper I, which is the reason for keeping the same notation and terminology ("apparent") for it.

\subsection{Local boxes, contrast definition and velocity calibration}

From the different AR and QS scans we extracted a series of square "boxes" containing different amounts of magnetic flux, all at $\mu>0.99$. In the network scans we found it useful to visually distinguish between "strong network", where the large magnetic patches are reminiscent of plages, and "weak network" containing rather isolated magnetic elements. Note that

\footnotetext{
1 This means that these quantities were inferred by taking into account all the light detected at the pixel, as if it were entirely coming from a magnetic atmosphere. These photons actually originate from the solar area sampled by the pixel (which can be only partially covered by magnetic fields) and from its local neighborhood (Orozco Suárez et al. 2007) due to diffraction by the relatively narrow point spread function of the Hinode/SOT (Danilovic et al. 2008).
} 
P. Kobel et al.: The continuum intensity as a function of magnetic field. II.

Table 1. List of the SP scans used in this work.

\begin{tabular}{lcccc}
\hline \hline Date (dd-mm-yy) & $t_{\text {start }}(\mathrm{UT})$ & Target & NOAA & No. boxes \\
\hline $11-12-06$ & $13: 10: 09$ & AR & 10930 & 3 \\
$05-01-07$ & $11: 20: 09$ & AR & 10933 & 5 \\
$01-02-07$ & $12: 14: 05$ & AR & 10940 & 5 \\
$28-02-07$ & $11: 54: 34$ & AR & 10944 & 2 \\
$01-05-07$ & $21: 00: 06$ & AR & 10953 & 7 \\
$11-05-07$ & $12: 35: 53$ & AR & 10955 & 3 \\
\hline $10-03-07$ & $11: 37: 36$ & QS & - & $5^{s} 2^{w}$ \\
$23-04-07$ & $11: 14: 06$ & QS & - & $5^{s} / 1^{w}$ \\
$24-04-07$ & $01: 21: 04$ & QS & - & $2^{s} / 1^{w}$ \\
$27-04-07$ & $08: 50: 03$ & QS & - & $4^{s} / 1^{w}$ \\
\hline
\end{tabular}

Notes. $t_{\text {start }}$ denotes the starting time of the scans, and "target" indicates whether the scan was performed over an active region (AR), or the quiet Sun (QS). The number of boxes ("No. boxes") extracted from each scan depended on the effective area of the field of view without sunspots and with $\mu>0.99$. ${ }^{(s)}$ Number of strong network boxes. ${ }^{(w)}$ Number of weak network boxes.

the strong network is similar to what has been called "enhanced network". The size of the boxes was a trade-off between having enough statistics to investigate the contrast of their magnetic elements, while being small enough to avoid mixing the three categories, namely plage, strong network and weak network. For the AR plage and the QS strong network we chose a box size of $20^{\prime \prime} \times 20^{\prime \prime}$. Due to the low concentration of flux and consequently poorer statistics in the weak network, we treated it in larger boxes of $70^{\prime \prime} \times 70^{\prime \prime}$. Note that most of the area of the weak network boxes is covered by internetwork fields. These contribute significantly to the total flux in these boxes. Also, it is now established that at least some of the magnetic features in the IN have $\mathrm{kG}$ field strengths and correspond to magnetic elements (Lagg et al. 2010; Sánchez Almeida et al. 2003). We selected a total of 25 plage boxes, 16 strong network boxes and 4 weak network/internetwork boxes. One example of each category is provided in Fig. 1 (where the weak network/internetwork boxes was cropped to an area of $20^{\prime \prime}$ for comparison).

We quantified the total unsigned magnetic flux in each box, $\Phi$, by the mean apparent longitudinal field strength $\left\langle B_{\text {app,los }}\right\rangle$, which relates to $\Phi$ by:

$\Phi=\int_{\text {box }} B_{\text {app,los }} \mathrm{d} A=\left\langle B_{\text {app,los }}\right\rangle A_{\text {box }}$,

where $A_{\text {box }}$ stands for the box area. $\left\langle B_{\text {app,los }}\right\rangle$ was chosen instead of $\Phi$ because the weak network boxes are larger than the strong network and plage ones. Thus, $\left\langle B_{\text {app,los }}\right\rangle$ represents the degree of concentration of the magnetic flux, or "magnetic activity", in the local area delimited by the box. Note that pores were included in the calculation of $\left\langle B_{\mathrm{app}, \mathrm{los}}\right\rangle$, as they also contribute to the inhibition of convection. However, as our study focuses mainly on the contrast of magnetic elements, pores and their immediate surroundings were removed from the subsequent contrast analysis using the same method as in Paper I (the yellow contours in the top left panel of Fig. 1 outline such pores).

In each box, the contrast at a pixel location $(x, y)$ was defined relative to the mean continuum intensity $\left\langle I_{c}\right\rangle_{\text {ref,box }}$ of all the pixels in the box having $B_{\text {app,los }}<100 \mathrm{G}$, which correspond to weakly magnetized areas harboring relatively normal granulation (see Fig. 1):

$\operatorname{Contrast}(x, y)=\frac{I_{\mathrm{c}}(x, y)-\left\langle I_{\mathrm{c}}\right\rangle_{\text {ref,box }}}{\left\langle I_{\mathrm{c}}\right\rangle_{\text {ref,box }}}$ using one contrast reference per box prevents the 5 min oscillations and possibly instrumental variations (e.g. varying amounts of defocus of the Hinode/SOT between scans, Danilovic et al. 2008) to introduce spurious scatter in the contrast data ${ }^{2}$.

Likewise, the LOS velocities $v_{\text {los }}$ were calibrated independently for each box, since oscillations can induce a global up- or downflow of an entire box, while we are interested in quantifying the local up- and downflows within the box. We thus calibrated the velocities in each box such that the average of $v_{\text {los }}$ over the areas where $B_{\text {app,los }}<100 \mathrm{G}$ is equal to the Doppler convective blueshift of the 630.2 line minimum as derived from the FTS spectrum at the spectral resolution of Hinode $\left(200 \mathrm{~m} \mathrm{~s}^{-1}\right)$.

\section{Results}

\subsection{Contrast of magnetic elements and surrounding flows}

In the first place we investigated how the contrast of magnetic elements varies with the amount of unsigned magnetic flux contained in the boxes. Therefore we made for each box a scatterplot of the continuum contrast vs. $B_{\text {app,los. We then proceeded as }}$ in Paper I, namely by averaging the pixel contrasts in $B_{\text {app,los}}{ }^{-}$ bins of $50 \mathrm{G}$ width, and fitting these average values by a 3rdorder polynomial (see Fig. 2). In the following we shall simply refer to the maximum of the polynomial trend as contrast peak, which can be considered as a measure of the (average) contrast of the brightest magnetic elements in the box. Figure 2 presents the scatterplots of the contrast vs. $B_{\text {app,los }}$ for the three boxes presented in Fig. 1, along with their trends and respective peaks (indicated by the arrows). Note how the trends vary between the three boxes: the peak increases from plage to weak network while the range of $B_{\text {app,los }}$ decreases. Together with the fact that the peak contrast occurs at $B_{\text {app,los }} \sim 700$ for all boxes, these observations are consistent with Paper I. Hence, despite the relatively small size of the boxes, they contain enough statistics to accurately determine the contrast peak. Note that for the weak network box, the trend is almost monotonically increasing. This is due to the absence of dark features such as micropores, which are common in the strong network and plages. Such quasimonotonic trends were also obtained by Schnerr \& Spruit (2011) and Viticchié et al. (2010), who considered fields of view containing QS network and internetwork, observed at SST and with IBIS, respectively.

Figure 3 a displays the relation between the contrast peak and $\left\langle B_{\text {app,los }}\right\rangle$ in the box. It can be seen that both quantities clearly anti-correlate, such that the magnetic elements are darker in the more active boxes. The relationship is linear for $\left\langle B_{\text {app,los }}\right\rangle>$ $50 \mathrm{G}$, but it cannot be excluded that the contrast of magnetic elements in regions with $\left\langle B_{\text {app,los }}\right\rangle \leq 50 \mathrm{G}$ might have a significantly stronger dependence on $\left\langle B_{\text {app,los }}\right\rangle$ than given by the regression line.

To test the idea that the convective energy transport surrounding the magnetic elements can affect their contrasts (see Sect. 1), we also determined the rms of the LOS velocity $v_{\text {los }}$ (returned by the inversions) as a measure of the vigour of the vertical convective flows in the boxes. Since we were only interested in characterizing the flows surrounding the magnetic features, we restricted the measurement of $\operatorname{rms}\left(v_{\text {los }}\right)$ to the pixels

2 This implicitely assumes that the intrinsic brightness of the weakly magnetized areas $B_{\text {app,los }}<100 \mathrm{G}$ does not vary from one box to the other. 

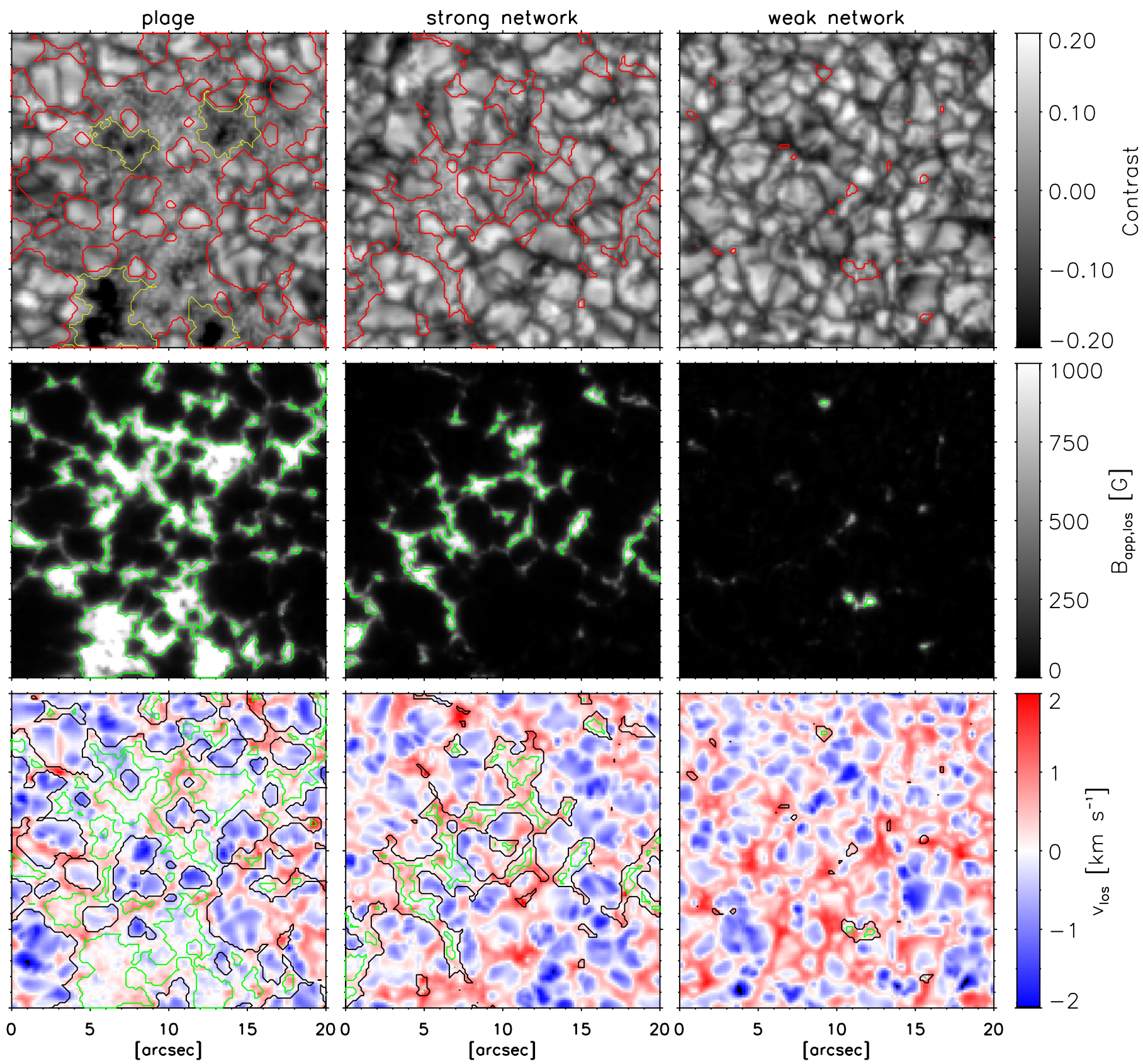

Fig. 1. Continuum contrast (top), apparent longitudinal field strength $B_{\text {app,los }}$ (middle) and LOS velocity $v_{\text {los }}$ (bottom) for three examples of boxes in active region plage (left), strong network (middle) and weak network (right), respectively. All the boxes are located at disk center $(\mu>0.99)$. The weak network box has been cropped to a subfield of $20^{\prime \prime} \times 20^{\prime \prime}$ for better visibility, although its original size is $70^{\prime \prime} \times 70^{\prime \prime}$. Yellow contours in top left: pores (see main text) excluded from the scatterplot of contrast vs. $B_{\text {app,los }}$ (Fig. 2) and from the averaged contrasts (Fig. 7). Red contours (top) and black contours (bottom): delimit the areas where $B_{\text {app,los }}>100 \mathrm{G}$ (magnetic patches) from the weakly magnetized areas whose mean intensity is used as reference for the contrast (see Eq. (2)). Green contours (middle and bottom): delimit the location of the pixels where $B_{\text {app,los }}>500 \mathrm{G}$, so that the region between black and green contours has $100 \mathrm{G}<B_{\text {app,los }}<500 \mathrm{G}$.

where $B_{\text {app,los }}<500 \mathrm{G}^{3}$. As can be seen from Fig. 2 (dashed vertical line), this relatively arbitrary threshold value is sufficiently far from the peak of the contrast vs. $B_{\text {app,los }}$ trends (occuring at $B_{\text {app,los }} \sim 700 \mathrm{G}$ ) to consider that those pixels do not belong to those magnetic elements producing the peak, nor to the stronger and darker magnetic features ${ }^{4}$. The locations

\footnotetext{
${ }^{3}$ It is already well-known that convection is strongly suppressed within the magnetic elements due to the $\mathrm{kG}$ strength of the field, see, e.g., the review of Schüssler (1992).

4 The same threshold was already adopted in Paper I.
}

where $B_{\text {app,los }}<500 \mathrm{G}$ are delimited by green contours in Fig. 1 (middle and bottom panels). In fact, pixels with $100<$ $B_{\text {app,los }}<500 \mathrm{G}$ correspond to abnormal granulation surrounding the magnetic features (see below). Their magnetic signal probably comes from the expanding canopies of the magnetic features (e.g. Solanki \& Steiner 1990) as well as a "bloom" of scattered polarization signal (Lites 2002). However, it cannot be excluded that such pixels also contain magnetic elements with small filling factors. Figure $3 \mathrm{~b}$ then reveals that the $\operatorname{rms}\left(v_{\mathrm{los}}\right)$ of the pixels with $B_{\text {app,los }}<500 \mathrm{G}$ linearly decreases with the $\left\langle B_{\text {app,los }}\right\rangle$ in the boxes. Note that the slope of the regression and 
P. Kobel et al.: The continuum intensity as a function of magnetic field. II.
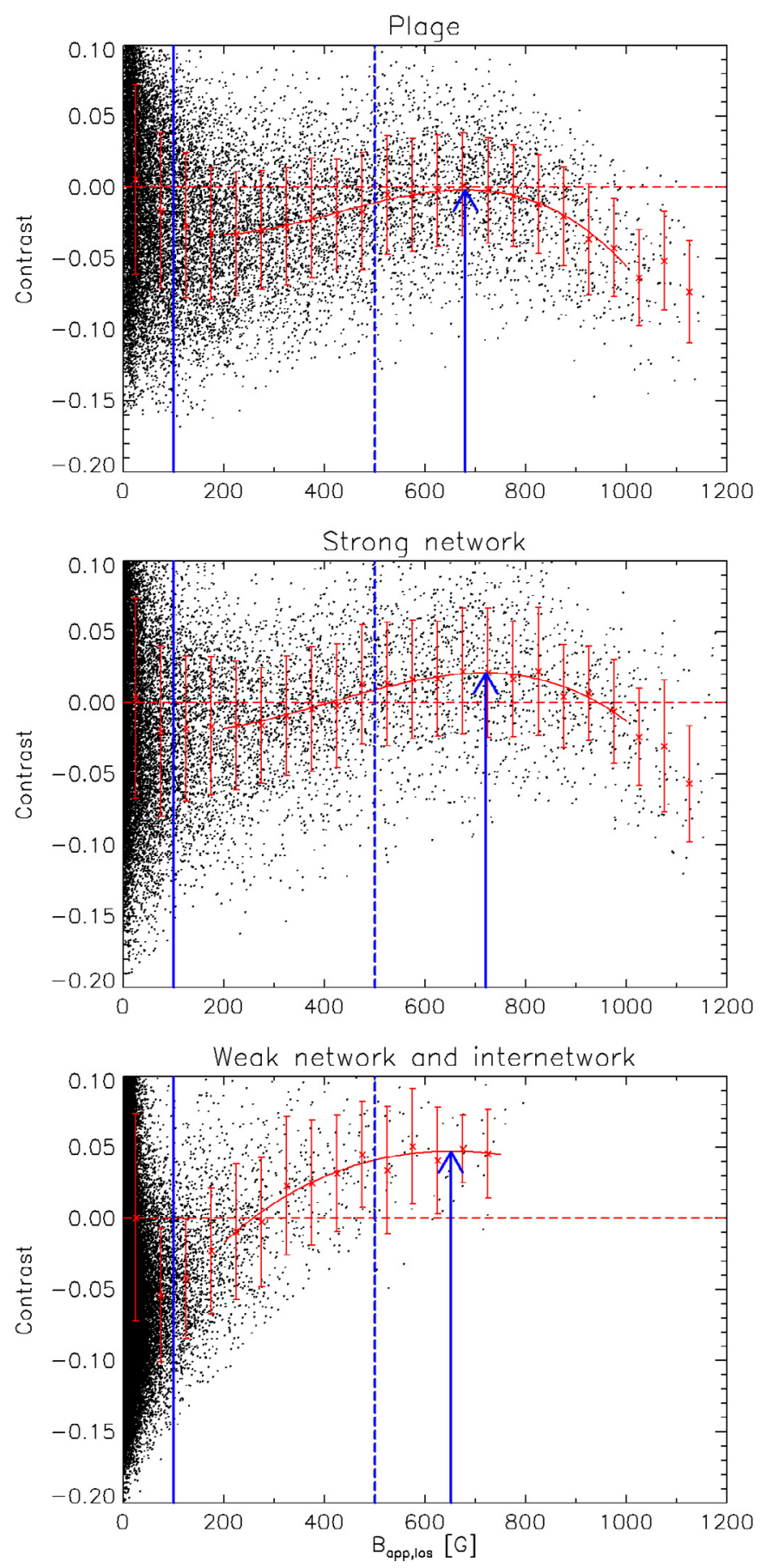

Fig. 2. Scatterplots of continuum contrast vs. apparent longitudinal field strength $B_{\text {app.los }}$ for the boxes shown in Fig. 1, pores excluded. Red crosses: average values of the continuum contrast inside $B_{\text {app,los }}$-bins of $50 \mathrm{G}$ width. The red error bars are the standard deviations inside each bin. Solid red curves are third-order polynomial fits of the average values in the range $200 \mathrm{G}<B_{\text {app,los }}<1000 \mathrm{G}$. Blue arrows: indicate

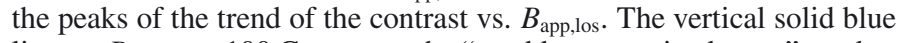
lines at $B_{\text {app,los }}=100 \mathrm{G}$ separate the "weakly magnetized areas" used as contrast and velocity reference and the "magnetic patches". The dashed blue lines at $B_{\text {app,los }}=500 \mathrm{G}$ is the upper limit below which pixels can be considered as not belonging to magnetic features.

correlation coefficient depend only weakly on the chosen upper limit of $B_{\text {app,los }}$ : if we chose a threshold of $300 \mathrm{G}$ instead, they would vary by less than $3 \%$. Note that, as a consequence of the anti-correlations of both the contrast peak and $\operatorname{rms}\left(v_{\text {los }}\right)$ with $\left\langle B_{\text {app,los }}\right\rangle$, the contrast peak correlates with $\operatorname{rms}\left(v_{\mathrm{los}}\right)$ (with a coeff. 0.79, plot not shown here). The decrease of both the contrast peak and $\operatorname{rms}\left(v_{\text {los }}\right)$ with $\left\langle B_{\text {app,los }}\right\rangle$ supports the idea that as the flux gets more concentrated in the boxes, the contrast of the magnetic elements is reduced as a result of a less efficient convection.

\subsection{Magnetic patches}

A closer look at the boxes gave us more insight as to how the embedded magnetic flux in the box can hamper the convection and affect the contrast of magnetic elements. Inside plages and strong network, most of the flux is contained in patches that have rather sharp boundaries (Title et al. 1992), also referred to as "magnetic islands" by Ishikawa et al. (2007) and "extended contiguous regions of strong field" by Narayan \& Scharmer (2010). In our case these areas can be relatively well enclosed by contours of $B_{\text {app,los }}>100 \mathrm{G}$ (see Fig. 1). These patches appear composed by clusters of small magnetic elements, micropores, pores and disturbed granulation (see also Sect. 4.2).

They contain between 60 and $95 \%$ of the total unsigned flux of the box, this fraction increasing rapidly with $\left\langle B_{\text {app,los }}\right\rangle$ as the patches cover a larger part of the box (see below). As can be seen from Fig. 4a, the total unsigned flux $\Phi$ (see Eq. (1)) contained in the box almost perfectly scales (correlation coeff. $>0.995$ ) with the total flux carried by the patches. Only the strong network and plage boxes have been represented because they have the same areas (hence their fluxes can be directly compared). Note that from the weak network to the plage boxes, the fractional area of the box covered by the patches varies by about one order of magnitude (from 0.05 to 0.55 ), whereas the average value of $B_{\text {app,los }}$ computed over the patches varies only between about 300 and $500 \mathrm{G}$ (see absissa of Fig. 5b) ${ }^{5}$. Hence, the amount of unsigned flux contained in a box, and thereby $\left\langle B_{\text {app,los }}\right\rangle$, is mainly determined by the fraction of the box occupied by these patches, as illustrated in Fig. 4b (with a correlation of 0.99). Since they carry most of the magnetic flux, we can attribute to the patches the degree of inhibition of vertical convection and the decrease of the contrast of the magnetic elements (see Sect. 4.2). To show this, we will separate the contributions from the patches from their weakly magnetized surroundings ( $\left.B_{\text {app,los }}<100 \mathrm{G}\right)$.

We shall first differentiate the state of convection inside and ouside the patches. Therefore, we evaluated $\operatorname{rms}\left(v_{\mathrm{los}}\right)$ and $\left\langle B_{\text {app,los }}\right\rangle$ separately in the areas outside $\left(B_{\text {app,los }}<100 \mathrm{G}\right)$ and inside the patches $\left(B_{\text {app,los }}>100 \mathrm{G}\right)$. For the latter, only the abnormal granulation within the patches was considered (with an upper limit of $500 \mathrm{G}$, cf. Sect. 3.1), while magnetic elements and pores were included in $\left\langle B_{\text {app,los }}\right\rangle$ (as both contribute to the inhibition of convection in their surroundings). The variation of $\operatorname{rms}\left(v_{\mathrm{los}}\right)$ as a function of $\left\langle B_{\text {app,los }}\right\rangle$ in the areas inside and outside magnetic patches is presented in Figs. 5a and b, respectively. Comparing the two figures, we see that the $\operatorname{rms}\left(v_{\text {los }}\right)$ in the patches is on average lower by about $110 \mathrm{~m} \mathrm{~s}^{-1}$, corresponding to a relative reduction of $\sim 21 \%$ (the average values of $\operatorname{rms}\left(v_{\text {los }}\right)$ are 527 and $417 \mathrm{~m} \mathrm{~s}^{-1}$ outside and inside the patches, respectively). That the $v_{\text {los }}$ amplitudes are reduced within the patches is also visible in the lower panels of Fig. 1, especially for the plage boxes. Note that the correlation in Fig. 5b is rather weak, mainly because $\left\langle B_{\text {app,los }}\right\rangle$ in the patch varies only between 300 and $550 \mathrm{G}$ (relative variation of $45 \%$ ). Also, the number of pixels satisfying $100<B_{\text {app,los }}<500 \mathrm{G}$ is relatively low, especially

5 The mean $B_{\text {app,los }}$ of the patches naturally slightly increases as they become larger, since there is more flux available for the formation of stronger and larger magnetic features. 
A\&A 542, A96 (2012)
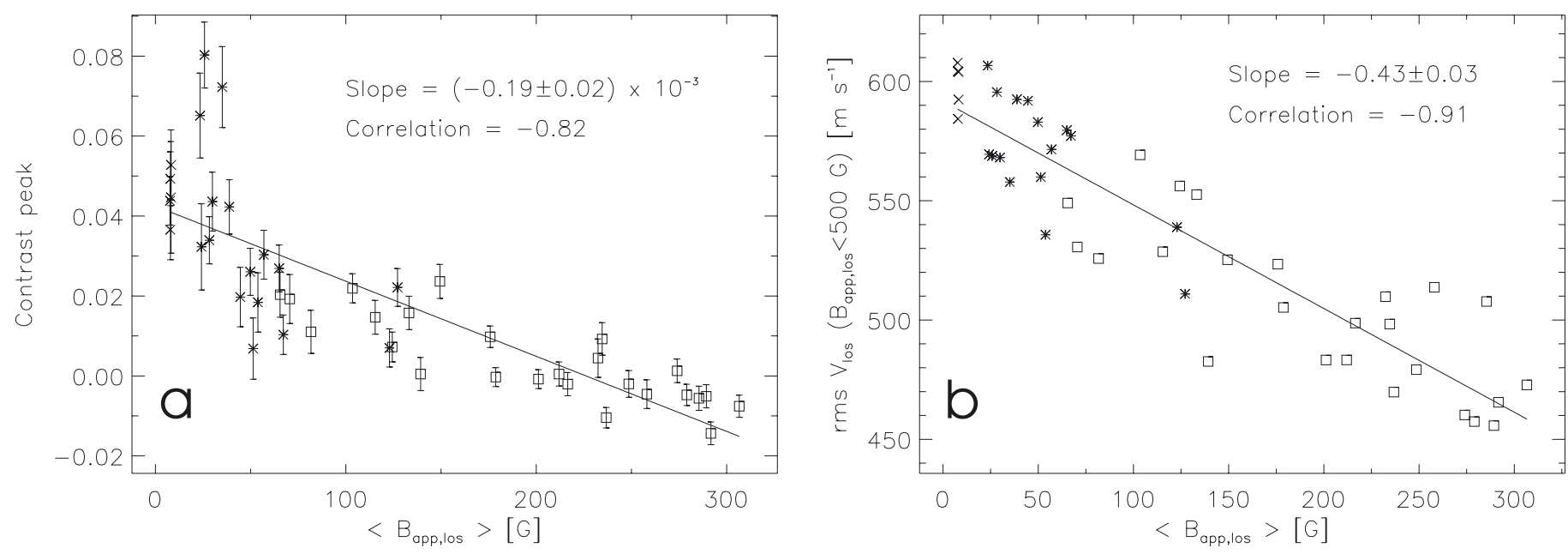

Fig. 3. a) Contrast peak vs. mean longitudinal field strength $\left\langle B_{\text {app.los }}\right\rangle$ for the plage (“" $\left.\square\right)$, strong network (“*”) and weak network boxes (“‘”). b) rms of the LOS velocity $v_{\text {los }}$ outside strong magnetic features $\left(B_{\text {app,los }}<500 \mathrm{G}\right)$ vs. $\left\langle B_{\text {app,los }}\right\rangle$ for the same boxes. Solid lines: linear regressions.
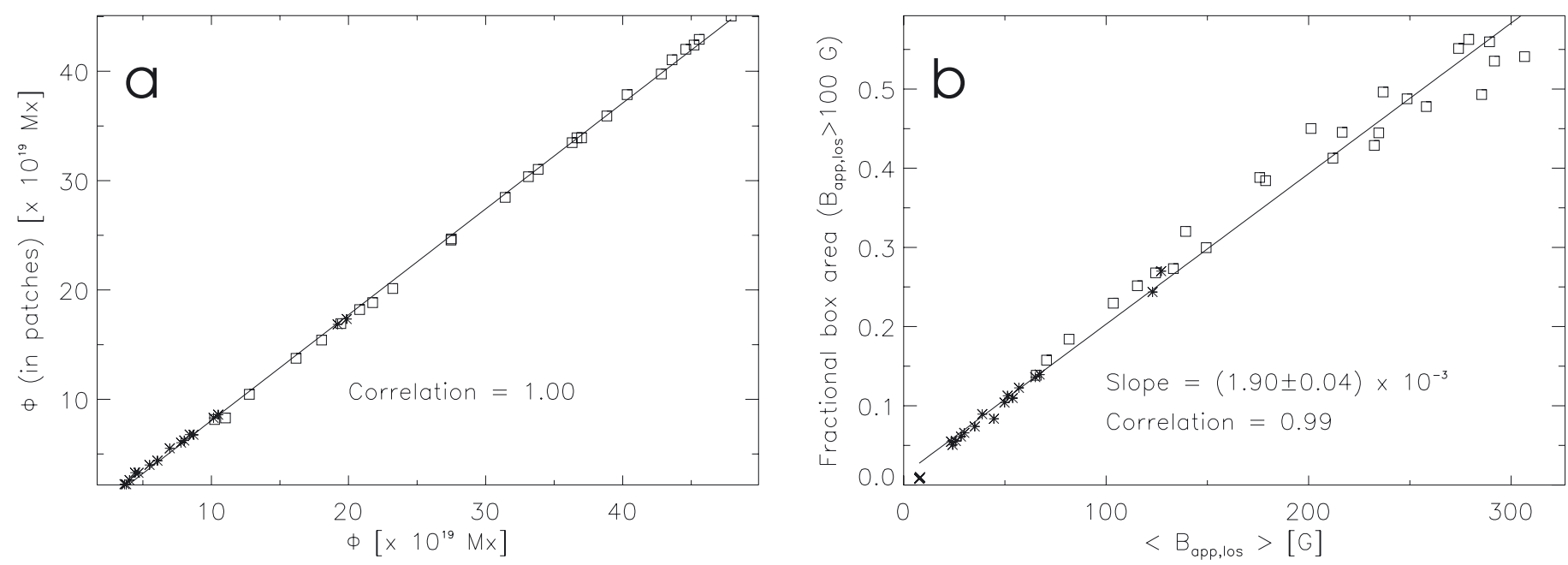

Fig. 4. a) Total unsigned magnetic flux carried by the magnetic patches ( $B_{\text {app.los }}>100 \mathrm{G}$ ) vs. total flux in the boxes for the strong network (“*”) and plage (" $\square$ ") boxes. b) Fraction of the box's area occupied by the magnetic patches vs. mean longitudinal field strength $\left\langle B_{\text {app,los }}\right\rangle$, for all the boxes including weak network (" $\times$ ").
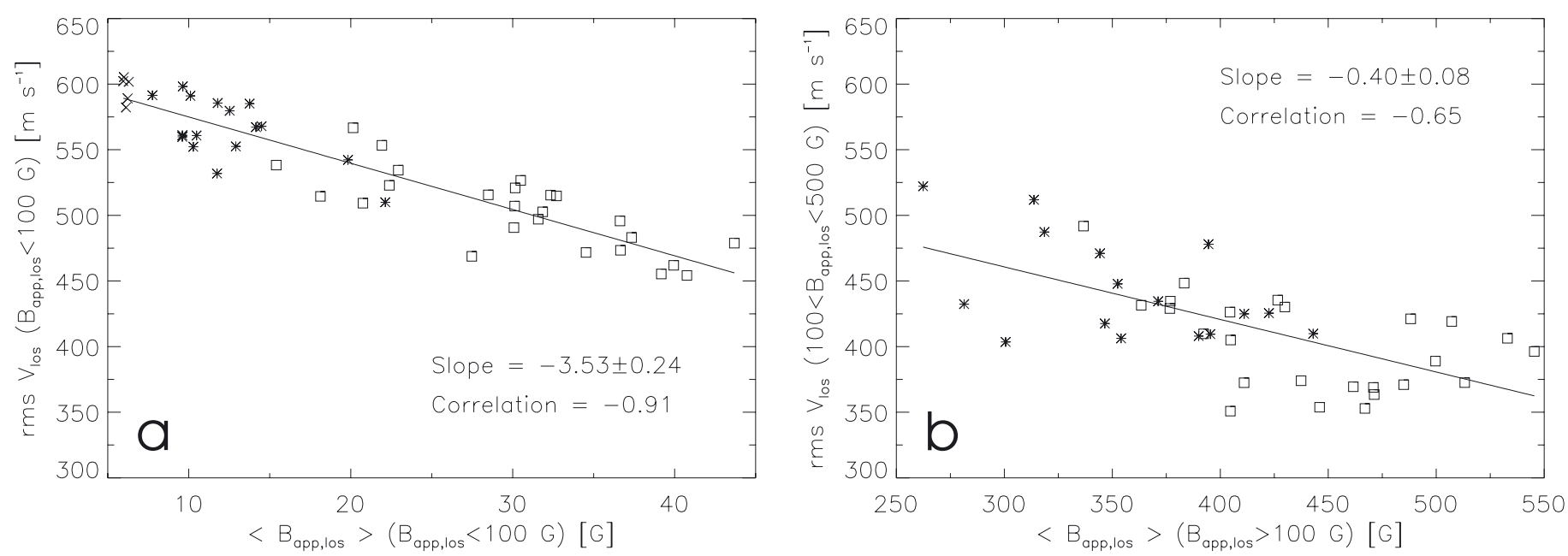

Fig. 5. a) rms of the LOS velocity $v_{\text {los }}$ vs. $\left\langle B_{\text {app,los }}\right\rangle$ outside the magnetic patches $\left(B_{\text {app,los }}<100 \mathrm{G}\right)$. b) rms of the LOS velocity $v_{\text {los }}$ vs. $\left\langle B_{\text {app,los }}\right\rangle$ inside the magnetic patches $\left(B_{\text {app,los }}>100 \mathrm{G}\right)$, but excluding magnetic features from the computation of $\operatorname{rms}\left(v_{\text {los }}\right)$ by restricting it to pixels with $B_{\text {app,los }}<500 \mathrm{G}$. 
P. Kobel et al.: The continuum intensity as a function of magnetic field. II.
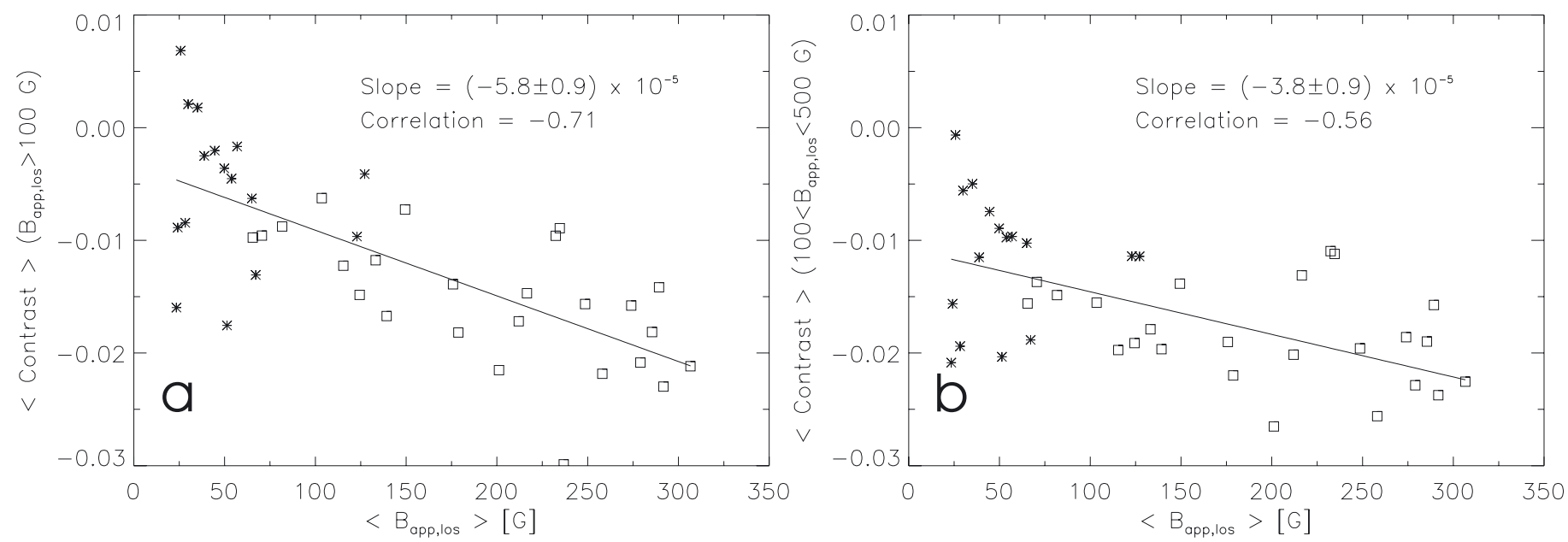

Fig. 6. a) Average contrast over the magnetic patches ( $B_{\text {app,los }}>100 \mathrm{G}$, pores removed) vs. mean longitudinal field strength in the boxes $\left\langle B_{\text {app,los }}\right\rangle$, for the strong network (“*”) and plage (“ $\square$ ”) boxes. b) Same but excluding magnetic features from the average contrast ( $\left.100<B_{\text {app,los }}<500 \mathrm{G}\right)$.

in the network boxes (these pixels cover only between 5 and $30 \%$ of the box area). In contrast, outside the patches $\left\langle B_{\text {app,los }}\right\rangle$ varies by a factor 4 , from 10 to $40 \mathrm{G}$, therefore the $\operatorname{rms}\left(v_{\text {los }}\right)$ exhibits a strong correlation $(-0.91)$ with $\left\langle B_{\text {app,los }}\right\rangle$ in these areas. As we shall see below (last paragraph and Fig. 8), however, the reduction of $\operatorname{rms}\left(v_{\mathrm{los}}\right)$ with $\left\langle B_{\text {app,los }}\right\rangle$ in these weakly-magnetized areas does not cause a reduction of their average brightness $\left\langle I_{\mathrm{c}}\right\rangle_{\text {ref,box }}$. The choice of $\left\langle I_{\mathrm{c}}\right\rangle_{\text {ref,box }}$ as contrast reference is thus also justified by its robustness with respect to variations of $\left\langle B_{\text {app,los }}\right\rangle$.

Next, we inquired whether the mean field strength in the boxes also affects the averaged contrast of the magnetic patches (outside the patches the contrast is equal to 0 by definition). Figure 6a displays the variation of the contrast averaged over the patches (pores excluded) as a function of $\left\langle B_{\text {app,los }}\right\rangle$ for all the boxes. Note that the weak network boxes were not included here because their patches are too small for proper statistics. Also, by our definition of contrast reference $\left\langle I_{\mathrm{c}}\right\rangle_{\text {ref,box }}$, the average contrast of the patches is equivalent to their average brightness relative to their weakly magnetized surroundings. As can be seen, the patches are always darker than their surrounds (except for 3 network boxes due to their bright magnetic elements), and become even darker as $\left\langle B_{\text {app,los }}\right\rangle$ increases and the patches grow bigger (i.e. occupy a larger fraction of the boxes cf. Fig. 4b). Since the magnetic patches contain both magnetic elements and abnormal granulation (pores being excluded from the analysis), we can further separate these two contributions. When averaging the contrast over the magnetic patches while at the same time excluding magnetic elements (i.e. considering only pixels with $100<B_{\text {app,los }}<500 \mathrm{G}$ ) as in Fig. $6 \mathrm{~b}$, we notice that the contrast values are now all negative and lower than in Fig. 6a, particularly for the QS network boxes from which we removed the bright magnetic elements (which are brighter than in plage boxes cf. Fig. 3a). This causes the slope of the regression line to decrease by a relative difference of $34 \%$. We can thus conclude that about one third of the darkening of the patches with increasing $\left\langle B_{\text {app,los }}\right\rangle$ can be attributed to the decreasing brightness of the magnetic elements, while about two thirds is due to a darkening of the abnormal granulation. This is understandable as the magnetic elements cover only between 10 and $40 \%$ of the area of the patches (with increasing fraction from network to plage boxes). In his 3D MHD simulations, Vögler (2005) similarly observed that both the decreasing intensity of the magnetic features and of the granulation were contributing to the darkening of the simulation snapshots (for $\langle B\rangle$ greater than $200 \mathrm{G}$ ).
Note that the correlations visible in Figs. 6a,b persist if plotting against the mean $B_{\text {app,los }}$ of the patch itself (although the correlation are reduced to -0.56 and -0.37 , respectively, but not shown here).

Finally, from the observation that the patches have negative contrast combined with the fact that they occupy a larger fractional area of the boxes as $\left\langle B_{\text {app,los }}\right\rangle$ increases, one can deduce that the contrast averaged over the entire boxes (pores excluded) will also exhibit a decreasing trend with $\left\langle B_{\text {app,los }}\right\rangle$, which is confirmed in Fig. 7. Note that the correlation coefficient $(-0.89)$ has improved compared to the correlation between the average contrast of the patches and $\left\langle B_{\text {app,los }}\right\rangle(-0.71$, cf. Fig. 6a). This is because the decrease of the box-averaged contrast with $\left\langle B_{\text {app,los }}\right\rangle$ is mainly dictated by the rapid increase of the fractional area of the patches with $\left\langle B_{\text {app,los }}\right\rangle$ (from 0.05 to 0.55 , cf. Fig. $4 b$ ), while the darkening of the patches with $\left\langle B_{\text {app,los }}\right\rangle$ contributes only marginally (it varies by about $1.5 \%$ only). In spite of the good correlation, the average box contrast decreases by only $\sim 1 \%$, as the average includes the areas of the box outside the patches, where the contrast is equal to 0 by definition. Hence, an area of photosphere in the $630 \mathrm{~nm}$ continuum at solar disk center containing strong network or plage appears darker than a quiet one, and its darkness increases with increasing magnetic flux.

To validate our contrast results, we checked that they were not biased by any systematic increase or decrease of the intrinsic brightness of the areas taken as contrast reference in each box (pixels with $B_{\text {app,los }}<100 \mathrm{G}$, see Sect. 2.2). A priori, the brightness of these areas could be affected by partial disturbances of convection (see the decrease of the $\operatorname{rms}\left(v_{\text {los }}\right)$ in Fig. 5a), enhanced radiative escape through a magnetized atmosphere, cooling by horizontal heat gradient toward the magnetic patches (Deinzer et al. 1984) and acoustic oscillations. To see if these effects induce any systematic trend, we compared in Fig. 8 the average intensity values $\left\langle I_{c}\right\rangle_{\text {ref,box }}$ (in "instrumental data numbers", i.e. units obtained after calibration with sp_prep, see Sect. 2.1) of the contrast reference areas in the boxes as a function of $\left\langle B_{\text {app,los }}\right\rangle$. As the weak correlation demonstrates, there is no systematic trend of $\left\langle I_{\mathrm{c}}\right\rangle_{\text {ref,box }}$ with $\left\langle B_{\text {app,los }}\right\rangle$, which validates the appropriate threshold of $100 \mathrm{G}$ for the contrast reference. As indicated by the right-hand side $y$-axis of the plot (where the values are normalized by the mean of all the $\left\langle I_{\mathrm{c}}\right\rangle_{\text {ref,box }}$ ), the linear regression varies by less than $0.5 \%$ over the range of $\left\langle B_{\text {app,los }}\right\rangle$. Hence, variations of the contrast reference from box to box cannot explain the decrease of $6 \%$ of the contrast peak (from 0.04 


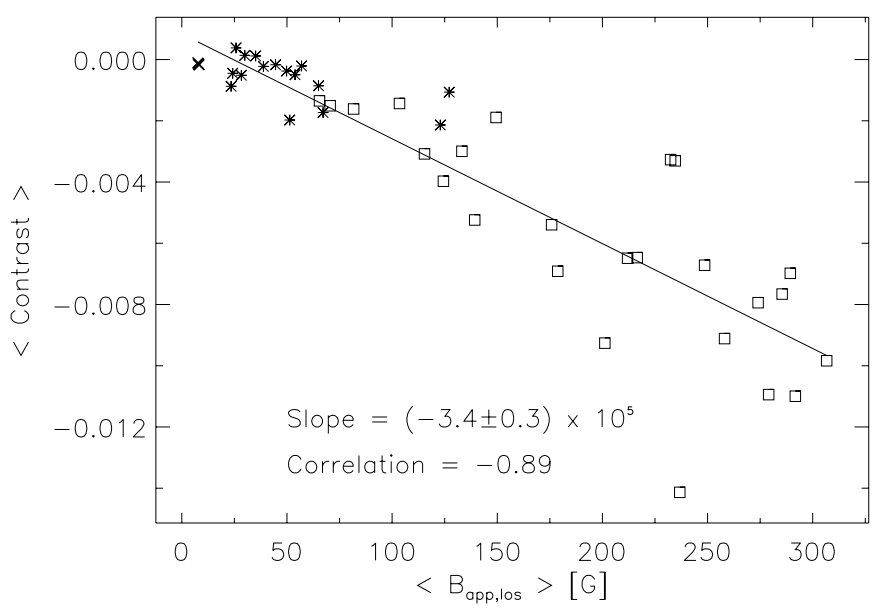

Fig. 7. Average of the contrast over the whole box (pores removed) vs. $\left\langle B_{\text {app,los }}\right\rangle$.

to -0.02 , see Fig. 3a) and the decrease of $\sim 1 \%$ of the boxaveraged contrast (Fig. 7) with $\left\langle B_{\text {app,los }}\right\rangle$. Note that the random fluctuations of $\left\langle I_{c}\right\rangle_{\text {ref,box }}$ can be attributed to oscillations and possibly to instrumental variations (cf. Sect. 2.2). Altogether, they produce a rms scatter of $1.3 \%$, much less than the scatter of the data points in Fig. 3a and of similar order than the scatter in Fig. 7. The relative stability of the contrast references enables us to interpret our contrasts as an indicator of the intrinsic brightness.

\section{Discussion}

\subsection{Dependence of the contrasts on the mean field strength}

Our box test showed that the contrast of the magnetic elements at disk center decreases continuously as the magnetic flux in the local environment defined by the box increases (see Fig. 3a). In particular, the absence of a gap between the network and the ARs implies that the physical mechanisms responsible for the brightness of the magnetic elements are primarily sensitive to the local mean field strength rather than to the elements' location within network or plage. According to the above, we expect the largest contrasts for nearly isolated bright points in weak network and internetwork regions.

At first sight, this result seems consistent with the conclusions of earlier spectra-based studies (e.g. Solanki \& Stenflo 1984; Zayer et al. 1990; Solanki \& Brigljevic 1992), which found on average darker magnetic features in ARs than in the QS. Although the studies based on Stokes profiles collect information originating solely from the magnetic features, their low spatial resolution (up to $10^{\prime \prime}$ in the case of FTS observations, Stenflo et al. 1984; Stenflo \& Harvey 1985) implies that the temperature information of magnetic elements may be mixed with that of micropores and pores also present in the resolution element. Hence, for these spectra-based studies the lower brightness in ARs can conceivably be due, in part or as a whole, to the presence of larger and darker magnetic features. An interpretation in terms of average magnetic feature size was also favored by Grossmann-Doerth et al. (1994), on the basis of radiationMHD models of magnetic features with different cross-sectional areas. The same conclusions were reached by Ortiz et al. (2002) using MDI data (angular resolution 4"). However, we believe that a variation of the size of the magnetic elements alone cannot explain our result, because we specifically looked at the peak

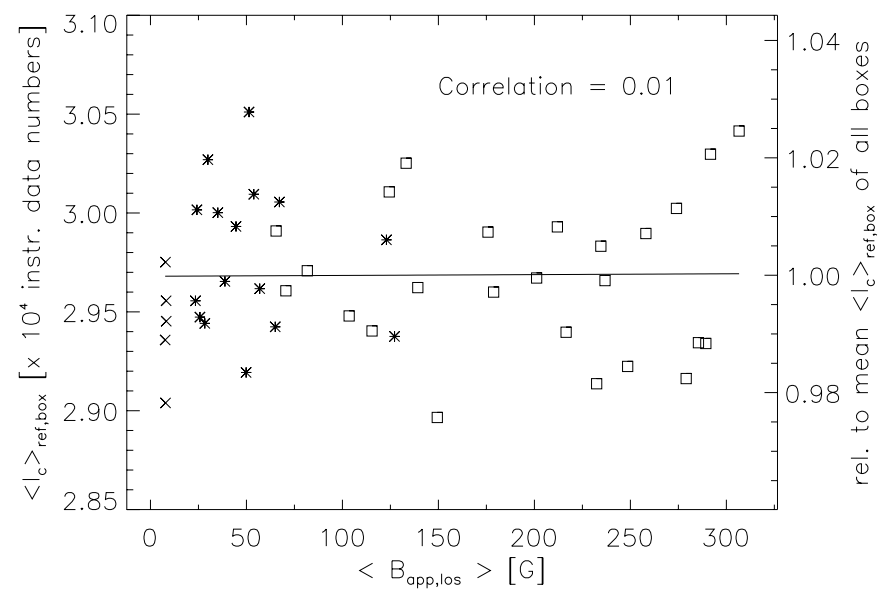

Fig. 8. Variation of the mean intensity $\left\langle I_{\mathrm{c}}\right\rangle_{\text {ref,FOV }}$ of the contrast reference areas in each box $\left(B_{\text {app,los }}<100 \mathrm{G}\right)$, as a function of $\left\langle B_{\text {app,los }}\right\rangle$ in the box. $\left\langle I_{\mathrm{c}}\right\rangle_{\mathrm{ref}, \mathrm{FOV}}$ is in instrumental data number. The solid line is a linear regression. The right-hand side $y$ axis gives the values relative to the mean value of all boxes.

of the contrast vs. $B_{\text {app,los }}$ trend in each box. As shown in Paper I (Fig. 3), the magnetic elements contributing to this peak share similar magnetic filling factors, which at Hinode's resolution can be assumed to scale with the cross-sectional area of the unresolved magnetic features.

This being said, one cannot exclude the possibility that the decrease of the contrast peak with $\left\langle B_{\text {app,los }}\right\rangle$ is indirectly influenced by the increasing number of larger features including micropores. Such features that could not be removed by our pore removal procedure are susceptible to have pixels with similar $B_{\text {app,los }}$ as the bright magnetic elements, especially toward their edges (see Paper I). The mixing of such relatively dark pixels with bright magnetic element pixels in the same $B_{\text {app,los }}{ }^{-}$ bin could then lower the contrast values. Telescope diffraction is also likely to play a role, as magnetic elements are often located in the direct vicinity of larger and darker flux concentrations (see Paper I Fig. 1). However, both these effects should lead to a larger dispersion in the scatterplots around the contrast peak (Fig. 2) for the AR boxes, which we do not observe.

To have an idea on the magnitude of these possible effects, it is instructive to look at how the number and area of larger magnetic features vary with the mean field in the boxes. Pores constitute a suitable target for that because they are generally sufficiently separated, so that their areas can be measured individually and are large enough to be easily segmented, even at Hinode's resolution. We thus quantified the number of pores and mean area of a pore (i.e. total area covered by the pores divided by the number of pores) in each of our strong network and plage boxes. To do so, we restricted the pores to their dark "cores" defined as the set of pixels where the contrast $<-0.15$ and $B_{\text {app,los }}>900 \mathrm{G}$ (cf. Paper I). As can be seen from Figs. 9a and $\mathrm{b}$, when $\left\langle B_{\text {app,los }}\right\rangle$ reaches $100-150 \mathrm{G}$, both the number and mean area (number of pixels per pore) increase rapidly with $\left\langle B_{\text {app,los }}\right\rangle$. If this is representative of the increasing number of larger-than-magnetic element features (not only pores), then it means that the effect of larger and darker features on the contrast of magnetic elements would only become significant for $\left\langle B_{\text {app,los }}\right\rangle>150 \mathrm{G}$, i.e. for the plage boxes.

Of direct relevance for the solar irradiance contributions near disk center is the decrease of the contrast averaged over the whole box as the magnetic flux increases. This result confirms and extends the finding of Title et al. (1992) that the mean 
P. Kobel et al.: The continuum intensity as a function of magnetic field. II.
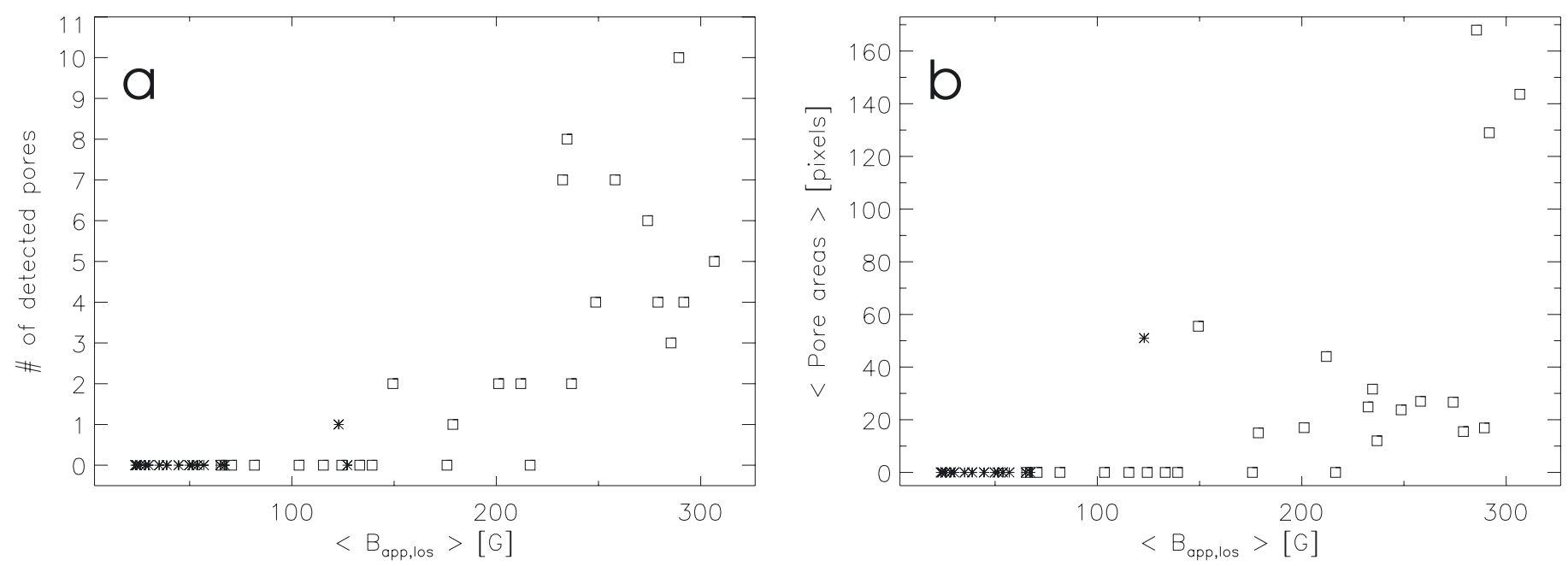

Fig. 9. a) Number of pores in the strong network (“*”) and plage (“口”) boxes as a function of $\left\langle B_{\text {app,los }}\right\rangle$. b) Mean area of the pores (in number of pixels) in each box vs. $\left\langle B_{\text {app,los }}\right\rangle$.

continuum contrast (at $676.8 \mathrm{~nm}$ ) of the single plage they observed (at $\mu=0.97$ ) was lower than the QS value. Although we only probed one wavelength, our result indicates a reduced vertical energy output with larger $\left\langle B_{\text {app,los }}\right\rangle$, as already predicted by the 3D MHD simulations of Vögler (2005). Note that the curve of mean bolometric intensity vs. mean field strength $\langle B\rangle$ of Vögler (2005) does not drop as linearly as ours and first exhibits an increase for $\langle B\rangle<100 \mathrm{G}$. In particular, the simulation boxes of Vögler (2005) yield mean bolometric intensities dropping below the QS level only for $\langle B\rangle$ greater than $200 \mathrm{G}$, whereas our box-averaged contrasts are negative even for mean longitudinal field strengths lower than $200 \mathrm{G}$ (see Fig. 7). These discrepancies can be attributed to different factors: an obvious one is that our measurements are monochromatic, so that they provide only a rough guide to the total energy loss, in particular that emitted at higher layers of the photosphere (e.g. in spectral lines). Another important factor is that the magnetic field in the simulations distributes itself more or less homogeneously in the intergranular lanes of the simulation box (i.e. in some ways like an infinitely large patch), whereas in the observations the magnetic elements tend to be clumped in patches, which are mainly responsible for the decrease in intensity and have a considerably higher average $B_{\text {app,los }}$ than the whole box. The cancellation of mixed polarities in our pixels and the fact that we only measure the longitudinal component of the field may also contribute. A visual comparison with the snapshots of Vögler (2005) also gives the impression that the magnetic patches within our boxes of strong network and plage (where we find negative average contrasts) rather resemble their box with $\langle B\rangle=400 \mathrm{G}$, for which the disk center bolometric intensity is lower than the QS value.

\subsection{Inhibition of convective flows}

The decrease of the contrast of the magnetic elements (Fig. 3a), the contrast of the patches (Fig. 6) and the box-averaged contrast (Fig. 7) with $\left\langle B_{\text {app,los }}\right\rangle$ could partly be caused by the inhibition of convection in the presence of magnetic fields. That the close packing of magnetic elements in plage could lead to a significant disturbance of the surrounding convective energy transport and thereby to lower contrasts was already proposed by Knölker \& Schüssler (1988), Knölker et al. (1988). This is supported by observational results from spectral lines indicating a weaker convective transport in regions with larger filling factors (Livingston 1982; Cavallini et al. 1985; Immerschitt \& Schröter 1987; Brandt \& Solanki 1990). Based on Hinode/SP data, Morinaga et al. (2008) recently gave evidence of the local action of the magnetic field on the flows, by observing that the vertical convection was more suppressed in locations (pixels) of larger apparent field strength. This is now validated on the larger scale of our boxes by showing that, as the amount of magnetic flux increases in the box, the convective flows surrounding the magnetic elements are progressively more suppressed (Fig. 3b).

Note that the linear relationship between $\operatorname{rms}\left(v_{\text {los }}\right)$ outside the magnetic elements and $\left\langle B_{\text {app,los }}\right\rangle$ is independent of the box size. We indeed repeated the analysis using boxes with side length varying from $10^{\prime \prime}$ to $70^{\prime \prime}$ centered at the same locations, and found that the slope of the linear regression did not vary by more than $5 \%$. This intuitively means that the convective flows are affected by the mean magnetic flux density and not by the total amount of flux.

Magnetic elements are not homogeneously distributed in the box, but located inside magnetic patches, which have been previously discussed by Ishikawa et al. (2007) and Narayan \& Scharmer (2010). Like us (see Fig. 5), they found that the dispersion of the vertical velocities is significantly reduced in the patches compared to their surroundings. In the boxes presented in Fig. 1, one can see that in the continuum (upper panels) the patches have a diffuse fluid-like appearance. The granulation therein can hardly be recognized while the vertical velocities (lower panels) appear significantly reduced compared to the surroundings. Both these effects are probably due to the high density of magnetic features in the patches, which locally hampers the convective motions (Fig. 5b) causing the spatial scale of convection to become smaller ("abnormal"). Telescope diffraction also contributes to the diffuse appearance of the patches. The combination of both effects could lead us to underestimate the $\operatorname{rms}\left(v_{\text {los }}\right)$ inside our patches, inasmuch as the velocity field might be partly under-resolved. With the help of SST CRISP data at 0 .'15 resolution, Narayan \& Scharmer (2010) were indeed able to identify a small-scale convection pattern within their patches and estimated its spatial scale to be $\sim 0$ ". 3 , i.e. similar to Hinode's resolution. Hence, as the amount of flux in the box increases, the reduced vertical velocities and reduced convection scale in the patches have the statistical consequence of decreasing the $\operatorname{rms}\left(v_{\text {los }}\right)$ in the box. 
The magnetically-disturbed convection within the patches (Fig. 5b) is also likely to be the cause of the reduced brightness of the abnormal granulation (Fig. 6b), just as deduced by Vögler (2005) from his simulations. The partial inhibition of convective motions and the reduced spatial scale of convection limit the convective heat upflow and the efficiency of the convective energy transport, which tends to cool the patch. Hence the patch becomes ever cooler and darker as its mean field strength and area increase, because both contribute to inhibit the convection. The inhibition of convection is driven by the patch size to a certain extent, since for a very small patch no granule is completely surrounded by magnetic features, while for a larger patch an increasing number of granules is. We speculate that the larger mean field strength of the patch is mostly due to an increased average size of magnetic features, which are then better resolved - i.e. have a larger magnetic filling factor - and due to the formation of somewhat stronger magnetic features. Since larger features tend to be darker, this is also likely to contribute to the darker appearance of the patch.

As magnetic elements are located inside the patches, their brightness should be affected by the local amount of heat available in the patch "reservoir". This would explain the decrease of the brightness of the magnetic elements from network to plage as the patches become larger and at the same time aquire a larger mean field. This interpretation is consistent with the finding of Ishikawa et al. (2007) that G-band bright points tend to lie preferentially close to the boundary of the magnetic patches, as the further inside they get, the farther they are from the heat source of normal granulation. Note that the fact that the magnetic patches appear darker than normal granulation combined with telescope diffraction could also play a role when comparing the observed contrasts of magnetic elements in ARs, which are mostly located within large patches, with the contrast of QS bright points, which are often isolated and surrounded by normal granulation (see Lawrence et al. 1993, and Paper I). The limited resolution of the telescope that blends the signals of the BPs could thus have a stronger darkening effect in ARs than in QS, although isolated QS BPs are also susceptible of being smeared out in the intergranular lanes (Title \& Berger 1996). However, we believe this is not the main effect causing the decrease of the contrast peak from the QS network to the AR plage boxes, as the mean contrast of the abnormal granulation in the patches (i.e. with $100 \mathrm{G}<\left\langle B_{\text {app,los }}\right\rangle<500 \mathrm{G}$ ) varies by only $\sim 1 \%$ over the range of $\left\langle B_{\text {app,los }}\right\rangle$ (Fig. 6b), whereas the contrast peak decreases by $\sim 6 \%$ (Fig. 3a).

\section{Summary and conclusions}

Previous studies found that at disk center, the continuum intensity contrast in the QS network reached higher values than in ARs (Title et al. 1989; Topka et al. 1992; Lawrence et al. 1993) and (Topka et al. 1997). This was confirmed by the first paper of this series (Kobel et al. 2011, Paper I) using Hinode/SP data, where we established that the relation between continuum brightness (at $630.2 \mathrm{~nm}$ ) and longitudinal field strength $B_{\text {app,los }}$ (retrieved by Milne-Eddington inversions) exhibits a higher peak in the QS network than in ARs. Since the magnetic elements producing the peak share similar magnetic filling factors, we argued that this brightness difference between the QS and ARs is unlikely to be explained solely by a difference in the size of the brightest magnetic elements (although the presence of larger features in ARs certainly plays a role in determining their brightness more generally, see below).
In this paper, we tested whether the contrast of the magnetic elements at disk center could be affected by an altered convective transport due to the local concentration of magnetic flux. With this aim, we extracted from Hinode/SP scans a series of "boxes" containing different amounts of magnetic flux, covering areas from AR plages to weak QS network. We found that the contrast of the brightest magnetic elements (i.e. the peak of the contrast vs. $\left.B_{\text {app,los }}\right)$ continuously decreases with the mean longitudinal field strength $\left\langle B_{\text {app,los }}\right\rangle$ in the boxes (Fig. 3a). Since the $\left\langle B_{\text {app,los }}\right\rangle$ is generally larger in ARs than in the QS, this can be taken as a generalization of the earlier finding that magnetic elements in the network are brighter than in ARs.

Hence, as the amount of flux (and thereby $\left\langle B_{\text {app,los }}\right\rangle$ ) increases in a local area, the observed contrast of the magnetic elements associated to the contrast peak decreases due to a combination of two effects: (i) the known presence of larger (and thus darker) magnetic features, even if pores are removed. These can either provide pixels. Such larger magnetic features reduce the contrast of nearby magnetic elements through the telescope point spread function. Yet this effect is likely to play only a minor role in the decrease of the magnetic element contrasts with $\left\langle B_{\text {app,los }}\right\rangle$ (see discussion in Sect. 4.1); (ii) the disturbance of convective energy transport in the surrounding of the magnetic features. This is supported by the anti-correlation between the fluctuation of the longitudinal velocities outside the magnetic features and $\left\langle B_{\text {app,los }}\right\rangle$ (Fig. 3b) and the lower brightness of the abnormal granulation surrounding magnetic elements compared to weakly magnetized areas (Fig. 6b).

A closer inspection of the strong network and plage boxes revealed that the magnetic flux in these boxes is mostly carried by "magnetic patches" that are well delimited by $B_{\text {app,los }}>100 \mathrm{G}$ (Fig. 4a). The flux density in these patches is such that the vertical convective velocities inside them (around magnetic features) are reduced significantly relative to their surroundings (the $\operatorname{rms}\left(v_{\text {los }}\right)$ is about $100 \mathrm{~m} \mathrm{~s}^{-1}$ lower), this reduction being more important as the mean field of the patches increases (cf. Fig. 5b). These reduced velocities probably limit the heat upflow and consequently lower the brightness of the granulation in the patches compared to the granulation in the weakly magnetized areas (Fig. 6b). The reduced spatial scale of the (abnormal) granulation inside the patches probably also contributes to decreasing the efficiency of the convective energy transport. Even with their magnetic elements, the patches are generally darker than their surroundings (Fig. 6a), and become all the more dark when $\left\langle B_{\text {app,los }}\right\rangle$ increases in the box and the patches occupy a larger part of it (Fig. 4b). We interpret the patches as having the following influence on the brightness of the magnetic elements: the more extended the magnetic patch is (independently of the box size) and the larger its mean field, the larger is the inhibition of convection, resulting in cooler granules and smaller heat influx to the "hot walls" of the magnetic elements which become cooler and less bright (at least at disk center).

Since the patches are darker than their surrounding and occupy a larger part of the box as $\left\langle B_{\text {app,los }}\right\rangle$ increases, the average brightness of a box at disk center also decreases with increasing $\left\langle B_{\text {app,los }}\right\rangle$ (by about $1.2 \%$ from weak network to plage boxes). Previous 3D MHD simulations of Vögler (2005) already predicted a reduction of the vertical bolometric intensity of boxes with mean field strength $\langle B\rangle>200 \mathrm{G}$. The qualitative agreement between our results and these simulations (for $\langle B\rangle>200 \mathrm{G}$ ) supports their use to model the different component atmospheres used in irradiance reconstructions (see, e.g., Unruh et al. 2009).

Up to now, the effect of the inhibition of convection on the photospheric contrast has not been taken into account in 
P. Kobel et al.: The continuum intensity as a function of magnetic field. II.

irradiance reconstruction models. These models only use the photometric area of magnetic features (Penza et al. 2003; Fontenla \& Harder 2005) or their "filling factor" within a pixel (in the case of SATIRE, see Solanki et al. 2005; Krivova et al. 2010 , for reviews) as the time-dependent variables modulating the intensity output given by semi-empirical 1D model atmospheres. Our results suggest that the reconstructions could be possibly enhanced by modulating the emitted intensity (e.g. for a given magnetic filling factor) according to the surrounding magnetic flux in a local (e.g. in a $20^{\prime \prime} \times 20^{\prime \prime}$ ) area. However, the finding that the inhibition of convection in more active areas affects the brightness of magnetic elements has been tested here for the disk center only. In the next article of this series, we shall investigate this effect away from the disk center by comparing center-to-limb variation of the continuum contrast of magnetic elements in the QS and in ARs separately.

Acknowledgements. We gratefully thank M. Schüssler, D. Rörbein, and the Solar Lower Atmosphere and Magnetism (SLAM) group at the Max-Planck Institut für Sonnensystemforschung, as well as J. de la Cruz Rodríguez, Y. Unruh, W. Ball, B. Viticchié, N. Krivova and J. Sanchez-Almeida for their interest and fruitful discussions about this work. This work has been partially supported by WCU grant No. R31-10016 funded by the Korean Ministry of Education Science and Technology. Hinode is a Japanese mission developed and launched by ISAS/JAXA, collaborating with NAOJ as a domestic partner, NASA and STFC (UK) as international partners. Scientific operation of the Hinode mission is conducted by the Hinode science team organized at ISAS/JAXA. This team mainly consists of scientists from institutes in the partner countries. Support for the post-launch operation is provided by JAXA and NAOJ (Japan), STFC (UK), NASA, ESA, and NSC (Norway). This work has also made use of the NASA ADS database.

\section{References}

Ball, W. T., Unruh, Y. C., Krivova, N. A., Solanki, S., \& Harder, J. W. 2011, A\&A, 530, A71

Beckers, J. M., \& Schröter, E. H. 1968, Sol. Phys., 4, 142

Borrero, J. M., Tomczyk, S., Kubo, M., et al. 2010, Sol. Phys., 35

Brandt, P. N., \& Solanki, S. K. 1990, A\&A, 231, 221

Cavallini, F., Ceppatelli, G., \& Righini, A. 1985, A\&A, 143, 116

Chapman, G. A., \& Sheeley, Jr., N. R. 1968, Sol. Phys., 5, 442

Danilovic, S., Gandorfer, A., Lagg, A., et al. 2008, A\&A, 484, L17

de Wijn, A. G., Rutten, R. J., Haverkamp, E. M. W. P., \& Sütterlin, P. 2005, A\&A, 441, 1183

de Wijn, A. G., Lites, B. W., Berger, T. E., et al. 2008, ApJ, 684, 1469

Deinzer, W., Hensler, G., Schüssler, M., \& Weisshaar, E. 1984, A\&A, 139, 435

Domínguez Cerdeña, I., Kneer, F., \& Sánchez Almeida, J. 2003, ApJ, 582, L55

Dunn, R. B., \& Zirker, J. B. 1973, Sol. Phys., 33, 281

Fligge, M., Solanki, S. K., \& Unruh, Y. C. 2000, A\&A, 353, 380

Fontenla, J., \& Harder, G. 2005, Mem. Soc. Astron. It., 76, 826

Foukal, P., \& Fowler, L. 1984, ApJ, 281, 442

Frazier, E. N., \& Stenflo, J. O. 1972, Sol. Phys., 27, 330

Fröhlich, C. 2000, Space Sci. Rev., 94, 15

Grossmann-Doerth, U., Knölker, M., Schüssler, M., \& Solanki, S. K. 1994, A\&A, 285, 648

Immerschitt, S., \& Schröter, E.-H. 1987, in The Role of Fine-Scale Magnetic Fields on the Structure of the Solar Atmosphere, ed. E.-H. Schröter, M. Vázquez, \& A. A. Wyller, 53

Ishikawa, R., Tsuneta, S., Kitakoshi, Y., et al. 2007, A\&A, 472, 911

Keller, C. U., \& Koutchmy, S. 1991, ApJ, 379, 751

Khomenko, E. V., Collados, M., Solanki, S. K., Lagg, A., \& Trujillo Bueno, J. 2003, A\&A, 408, 1115
Knölker, M., \& Schüssler, M. 1988, A\&A, 202, 275

Knölker, M., Schüssler, M., \& Weisshaar, E. 1988, A\&A, 194, 257

Kobel, P., Solanki, S. K., \& Borrero, J. M. 2011, A\&A, 531, A112

Krivova, N. A., Solanki, S. K., Fligge, M., \& Unruh, Y. C. 2003, A\&A, 399, L1

Krivova, N. A., Solanki, S. K., \& Unruh, Y. C. 2010, J. Atm. Sol.-Terr. Phys., in press

Lagg, A., Solanki, S. K., Riethmüller, T. L., et al. 2010, ApJ, 723, L164

Lawrence, J. K. 1988, Sol. Phys., 116, 17

Lawrence, J. K., Topka, K. P., \& Jones, H. P. 1993, J. Geophys. Res., 98, 18911

Lin, H. 1995, ApJ, 446, 421

Lites, B. W. 2002, ApJ, 573, 431

Livingston, W. C. 1982, Nature, 297, 208

Morinaga, S., Sakurai, T., Ichimoto, K., et al. 2008, A\&A, 481, L29

Muller, R. 1983, Sol. Phys., 85, 113

Muller, R., \& Keil, S. L. 1983, Sol. Phys., 87, 243

Muller, R., \& Roudier, T. 1984, Sol. Phys., 94, 33

Narayan, G., \& Scharmer, G. B. 2010, A\&A, 524, A3

Orozco Suárez, D., Bellot Rubio, L. R., Del Toro Iniesta, J. C., et al. 2007, PASJ, 59,837

Ortiz, A., Solanki, S. K., Domingo, V., Fligge, M., \& Sanahuja, B. 2002, A\&A, 388, 1036

Penza, V., Caccin, B., Ermolli, I., Centrone, M., \& Gomez, M. T. 2003, in Solar Variability as an Input to the Earth's Environment, ed. A. Wilson, ESA SP, 535, 299

Rabin, D. 1992, ApJ, 391, 832

Riethmüller, T. L., Solanki, S. K., Martínez Pillet, V., et al. 2010, ApJ, 723, L169 Rüedi, I., Solanki, S. K., Livingston, W., \& Stenflo, J. O. 1992, A\&A, 263, 323 Sánchez Almeida, J., Domínguez Cerdeña, I., \& Kneer, F. 2003, ApJ, 597, L177 Schmidt, W., Grossmann-Doerth, U., \& Schroeter, E. H. 1988, A\&A, 197, 306 Schnerr, R. S., \& Spruit, H. C. 2011, A\&A, 532, A136

Schüssler, M. 1992, in NATO Advanced Study Institute Series C Proc. 373: The Sun: A Laboratory for Astrophysics, ed. J. T. Schmelz, \& J. C. Brown, 191 Schüssler, M., \& Solanki, S. K. 1988, A\&A, 192, 338

Sheeley, Jr., N. R. 1969, Sol. Phys., 9, 347

Solanki, S. K. 1993, Space Sci. Rev., 63, 1

Solanki, S. K., \& Brigljevic, V. 1992, A\&A, 262, L29

Solanki, S. K., \& Fligge, M. 2002, Adv. Space Res., 29, 1933

Solanki, S. K., \& Steiner, O. 1990, A\&A, 234, 519

Solanki, S. K., \& Stenflo, J. O. 1984, A\&A, 140, 185

Solanki, S. K., Zufferey, D., Lin, H., Rüedi, I., \& Kuhn, J. R. 1996, A\&A, 310, L33

Solanki, S. K., Krivova, N. A., \& Wenzler, T. 2005, Adv. Space Res., 35, 376

Solanki, S. K., Inhester, B., \& Schüssler, M. 2006, Rep. Prog. Phys., 69, 563

Spruit, H. C. 1976, Sol. Phys., 50, 269

Spruit, H. C., \& Zwaan, C. 1981, Sol. Phys., 70, 207

Steiner, O. 2007, in Modern Solar Facilities - Advanced Solar Science, ed. F. Kneer, K. G. Puschmann, \& A. D. Wittmann (Universitätsverlag Göttingen), 321

Stenflo, J. O. 1973, Sol. Phys., 32, 41

Stenflo, J. O., \& Harvey, J. W. 1985, Sol. Phys., 95, 99

Stenflo, J. O., Solanki, S., Harvey, J. W., \& Brault, J. W. 1984, A\&A, 131, 333

Suematsu, Y., Tsuneta, S., Ichimoto, K., et al. 2008, Sol. Phys., 249, 197

Tarbell, T. D., \& Title, A. M. 1977, Sol. Phys., 52, 13

Title, A. M., \& Berger, T. E. 1996, ApJ, 463, 797

Title, A. M., Tarbell, T. D., Topka, K. P., et al. 1989, ApJ, 336, 475

Title, A. M., Topka, K. P., Tarbell, T. D., et al. 1992, ApJ, 393, 782

Topka, K. P., Tarbell, T. D., \& Title, A. M. 1992, ApJ, 396, 351

Topka, K. P., Tarbell, T. D., \& Title, A. M. 1997, ApJ, 484, 479

Tsuneta, S., Ichimoto, K., Katsukawa, Y., et al. 2008, Sol. Phys., 249, 167

Unruh, Y. C., Solanki, S. K., Schüssler, M., Vögler, A., \& Garcia-Alvarez, D. 2009, in AIP Conf. Ser. 1094, ed. E. Stempels, 768

Viticchié, B., Del Moro, D., Criscuoli, S., \& Berrilli, F. 2010, ApJ, 723, 787

Vögler, A. 2005, Mem. S.A.It., 76, 842

Vögler, A., \& Schüssler, M. 2003, Astron. Nachr., 324, 399

Wenzler, T., Solanki, S. K., Krivova, N. A., \& Fröhlich, C. 2006, A\&A, 460, 583

Zayer, I., Stenflo, J. O., Keller, C. U., \& Solanki, S. K. 1990, A\&A, 239, 356 